\title{
The use of dual reciprocity method for 2D laminar viscous flow
}

\author{
Juraj Mužík ${ }^{1, *}$ \\ ${ }^{1}$ University of Žilina, Faculty of civil engineering, Univerzitná 8215/1 01026 Žilina, Slovakia
}

\begin{abstract}
The paper presents the use of the dual reciprocity multidomain singular boundary method (SBMDR) for the solution of the laminar viscous flow problem described by Navier-Stokes equations. A homogeneous part of the solution is solved using a singular boundary method with the 2D Stokes fundamental solution - Stokeslet. The dual reciprocity approach has been chosen because it is ideal for the treatment of the nonhomogeneous and nonlinear terms of Navier-Stokes equations. The presented SBMDR approach to the solution of the $2 \mathrm{D}$ flow problem is demonstrated on a standard benchmark problem - lid-driven cavity.
\end{abstract}

\section{Introduction}

The solution of Navier-Stokes (NS) equations is one of the basic tasks of computational fluid mechanics. This nonlinear system of differential equations has already been solved by a number of numerical methods, starting with the finite difference method through the finite element method to meshless and boundary type methods. The methods based on boundary integral theory are represented by the local boundary integral element method (LBIEM) [1], the boundary element method (BEM) [2], [3], the method of fundamental solutions (MFS) [4] and the singular boundary method (SBM) [5]. In the case of BEM the singularities of the fundamental solution are handled by proper integration method, the MFS overcomes the singularity using a fictitious boundary, but the optimum location of this boundary remains the open problem especially for complex-shaped domains. To bypass the fictitious boundary construction, the SBM formulation adopts a concept of the origin intensity factors (OIFs). Several techniques have been developed to determine the source intensity factors, namely, inverse interpolation technique (IIT), subtracting and adding-back regularization and empirical formulas [5], [6].

In this article, the solution of the steady fluid flow governed by the primitive variable form of Navier-Stokes (NS) equations is presented. The SBM with dual reciprocity (DR) technique is used to handle the nonlinear convection terms of NS equations.

\footnotetext{
* Corresponding author: muzik@fstav.uniza.sk
} 


\section{Governing equations}

Incompressible steady flow is governed by Navier-Stokes equations in the closed domain $\Omega$ with the boundary $\Gamma$, which can be written in their primitive variables as

$$
\begin{gathered}
\frac{\partial u_{i}}{\partial x_{i}}=0 \\
\mu \frac{\partial^{2} u_{i}}{\partial x_{j} \partial x_{j}}-\frac{\partial p}{\partial x_{i}}=\rho \frac{\partial}{\partial x_{j}}\left(u_{j} u_{i}\right)+f_{i}
\end{gathered}
$$

where $u_{i}$ is the velocity vector component in the direction $i, p$ is the pressure, $v$ is the kinematic viscosity, $\rho$ is the density of a liquid, and $f_{i}$ represents body forces components. The boundary value problem describing fluid flow should satisfy boundary conditions in the form

$$
\begin{aligned}
& u_{i}\left(x_{D}\right)=f_{i}\left(x_{D}\right) x_{D} \in \Gamma_{D} \\
& t_{i}\left(x_{N}\right)=\sigma_{i j} n_{j}\left(x_{N}\right) x_{N} \in \Gamma
\end{aligned}
$$

where $u_{i}$ are the velocities and $t_{i}$ are the tractions on the boundary and $n_{j}$ represents the outward normal to the boundary of the computational domain. The stress tensor for Newtonian fluid is defined as

$$
\sigma_{i j}=-p \delta_{i j}+\mu\left(\frac{\partial u_{i}}{\partial x_{j}}+\frac{\partial u_{j}}{\partial x_{i}}\right)
$$

The solution of (1) and (2) in the context of dual reciprocity and singular boundary method, can be defined as the sum

$$
u_{i}=u_{i}^{\mathrm{H}}+u_{i}^{\mathrm{P}}, p=p^{\mathrm{H}}+p^{\mathrm{P}}
$$

where $u^{\mathrm{H}}$ are the solutions of the homogeneous problem that captures the Stokes equations together with the boundary conditions (3), (4) and $u^{\mathrm{P}}{ }_{\mathrm{i}}$ and $p^{\mathrm{P}}$ are the particular solutions of the non-homogeneous equations (1) and (2). The homogeneous problem can be determined using the singular boundary method (SBM) and the particular solution is formulated using the method of the particular solution (MAPS).

The singular boundary method (SBM) is based on the same principle as the method of fundamental solutions (MFS) however the SBM utilizes the same set of collocation and source points placed on the boundary $\Gamma$. The Green's function $G_{i j}$ is the fundamental solution of the Stokes equation known as Stokeslet

$$
G_{i j}(x, y)=-\frac{1}{4 \pi}\left(\ln \left(\frac{1}{|x-y|}\right) \delta_{i j}+\frac{\left(x_{i}-y_{i}\right)\left(x_{j}-y_{j}\right)}{|x-y|^{2}}\right)
$$

The Stresslet fundamental solution $K_{i j}$ is

$$
K_{i j}(x, y)=-\frac{1}{\pi} \frac{\left(x_{k}-y_{k}\right)\left(x_{i}-y_{i}\right)\left(x_{j}-y_{j}\right)}{|x-y|^{4}} n_{j}(y)
$$

and corresponding pressure solution $p_{j}$ is 


$$
p_{j}(x, y)=-\frac{1}{2 \pi} \frac{\left(x_{j}-y_{j}\right)}{|x-y|^{2}}
$$

The singular boundary method defines the solution of the homogeneous problem using the Green's functions as

$$
\begin{aligned}
& u_{i}^{H}\left(x^{m}\right)=\sum_{k=1, k \neq m}^{n} \alpha_{k} G_{i j}\left(x^{m}, y^{k}\right)+\alpha_{m} U_{i j}\left(x^{m}, y^{m}\right) \\
& t_{i}^{H}\left(x^{m}\right)=\sum_{k=1, k \neq m}^{n} \alpha_{k} K_{i j}\left(x^{m}, y^{k}\right)+\alpha_{m} T_{i j}\left(x^{m}, y^{m}\right)
\end{aligned}
$$

where $\alpha_{k}$ are the unknown coefficients, $x^{m}$ and $y^{k}$ are the collocation and source points and $U_{i j}, T_{i j}$ are the origin intensity factors, respectively. The key problem in SBM formulation is the way how the origin intensity factors (OIFs) are determined.

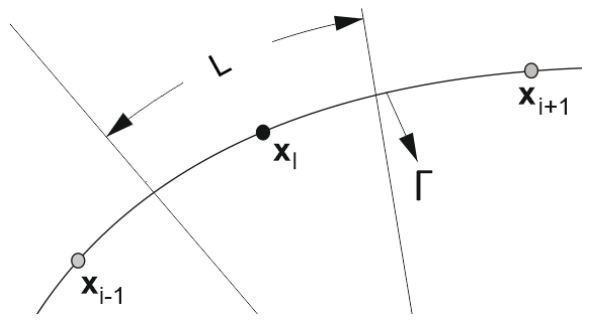

Fig. 1. Part of boundary $\Gamma$ around point $\mathbf{x}_{i}$.

The OIFs associated with the Stresslet and Stokeslet fundamental solution for the Neumann type boundary condition (pressure and velocity derivative) can be calculated using subtracting and adding-back technique [7], [8]

$$
T_{i j}\left(x^{m}, y^{m}\right)=-\frac{1}{L_{m}} \sum_{k=1, k \neq m}^{n} L_{k} \frac{\partial G_{i j}\left(x^{m}, y^{k}\right)}{\partial n\left(y^{k}\right)}
$$

where $L_{j}$ is the length of the appropriate part of the boundary around point $\mathbf{x}$ (see Fig.1). In the case of the Dirichlet boundary condition, the inverse interpolation technique (IIT) has been adopted for the OIFs associated with Neumann boundary conditions (prescribed velocities and pressures). IIT uses any existing particular solution for the Stokes flow problem. This particular solution can be constructed using the approach described in section 3 . The computational domain is covered by a set of scattered points at which the particular solution is known. The following system of equations (13) is then solved to obtain the unknown OIFs values.

$$
\left(\begin{array}{ccccc}
G_{i j}\left(\mathbf{y}_{1}, \mathbf{x}_{1}\right) & G_{i j}\left(\mathbf{y}_{1}, \mathbf{x}_{2}\right) & \cdots & G_{i j}\left(\mathbf{y}_{1}, \mathbf{x}_{k}\right) & \mathbf{1} \\
G_{i j}\left(\mathbf{y}_{2}, \mathbf{x}_{1}\right) & G_{i j}\left(\mathbf{y}_{2}, \mathbf{x}_{2}\right) & \cdots & G_{i j}\left(\mathbf{y}_{2}, \mathbf{x}_{k}\right) & \mathbf{1} \\
\cdots & \cdots & \ddots & \cdots & \cdots \\
G_{i j}\left(\mathbf{y}_{k}, \mathbf{x}_{1}\right) & G_{i j}\left(\mathbf{y}_{k}, \mathbf{x}_{2}\right) & \cdots & G_{i j}\left(\mathbf{y}_{k}, \mathbf{x}_{k}\right) & \mathbf{1} \\
\mathbf{1} & \mathbf{1} & \cdots & \mathbf{1} & \mathbf{0}
\end{array}\right)\left(\begin{array}{c}
C_{i j}^{1} \\
C_{i j}^{2} \\
\cdots \\
C_{i j}^{k} \\
\boldsymbol{\gamma}
\end{array}\right)=\left(\begin{array}{c}
U_{i j}^{1}\left(\mathbf{y}_{1}\right) \\
U_{i j}^{2}\left(\mathbf{y}_{2}\right) \\
\cdots \\
U_{i j}^{k}\left(\mathbf{y}_{k}\right) \\
\mathbf{0}
\end{array}\right)
$$

from which the unknowns $\mathrm{C}_{\mathrm{ij}}^{\mathrm{k}}$ and $\boldsymbol{\gamma}$ are solved. Using the computed intensity factors the for the nodes inside the domain, the OIFs for boundary nodes can be obtained, find more details in [6]. 


\section{Dual reciprocity method - Particular solution}

The particular solutions $u^{P}$, and $p^{P}$ of the (6) is approximated using a linear super position of the radial basis functions [2]

$$
u_{i}^{\mathrm{P}}=\sum_{j=1}^{M} \beta_{j k} \bar{u}_{i k}\left(r_{j}\right), p^{\mathrm{P}}=\sum_{j=1}^{M} \beta_{j k} \bar{p} k\left(r_{j}\right)
$$

where $M$ is the number of the internal points, $\beta_{j}$ are the unknown coefficients, and $\bar{u}_{i k}$ and $\bar{p}_{k}$ are the radial basis functions which can be defined as the particular solutions of the following non-homogeneous system of equations [2], [9]

$$
\begin{gathered}
\mu \frac{\partial^{2} \bar{u}_{i k}}{\partial x_{j} \partial x_{j}}-\frac{\partial \bar{p}_{k}}{\partial x_{i}}=\varphi(r) \delta_{i k} \\
\frac{\partial \bar{u}_{i k}}{\partial x_{i}}=0
\end{gathered}
$$

where $\varphi(r)$ is the thin plate spline (TPS) radial basis function (RBF) defined as

$$
\varphi(r)=r^{2} \log (r)
$$

where $r$ is Euclidean distance from the function.

One of the main difficulties is how to find a closed-form expression of the particular solutions $\bar{u}_{i k}$ and $\bar{p}_{k}$. To solve the problem, we employ the way more detailed described in [9]. As the first step, an auxiliary potential $\Phi$ is defined and is used to express the velocity particular solution as follows

$$
\bar{u}_{i k}=\frac{\partial^{2} \Phi}{\partial x_{j} \partial x_{j}} \delta_{i k}-\frac{\partial^{2} \Phi}{\partial x_{i} \partial x_{k}}
$$

The velocity particular solution captures implicitly the continuity equation, which can be checked by supplying the above velocity field into the continuity equation. Substituting (17) into the momentum equation (15) allows us to write

$$
\mu\left(\frac{\partial^{4} \Phi}{\partial x_{j} \partial x_{j} \partial x_{k} \partial x_{k}} \delta_{i k}-\frac{\partial^{4} \Phi}{\partial x_{j} \partial x_{j} \partial x_{i} \partial x_{k}}\right)-\frac{\partial \bar{p}_{k}}{\partial x_{i}}=\varphi \delta_{i k}
$$

The particular solution we explore is represented by the RBF approximation of the auxiliary potential used to express velocity, pressure and stress fields. The particular solution can be found splitting the momentum equation (18) into two parts and satisfying these two equations. Then the following system is obtained

$$
\begin{gathered}
\mu \frac{\partial^{4} \Phi}{\partial x_{j} \partial x_{j} \partial x_{k} \partial x_{k}}=\varphi(r) \\
\mu \frac{\partial^{4} \Phi}{\partial x_{j} \partial x_{j} \partial x_{i} \partial x_{k}}+\frac{\partial \bar{p}_{k}}{\partial x_{i}}=0
\end{gathered}
$$

The second equation (20) can be used to obtain pressure particular solution 


$$
\bar{p}_{k}=\mu \frac{\partial^{3} \Phi}{\partial x_{j} \partial x_{j} \partial x_{k}}
$$

The first equation (19) is used to compute the auxiliary potential $\Phi$ by the direct integration

$$
\Phi(r)=\frac{r^{6} \log (r)}{576}-\frac{5}{6} \frac{r^{6}}{576}
$$

Substituting the auxiliary potential $\Phi$ to the equations (17) and (21) the pressure and velocity field of a particular solution is obtained. The numerical value of viscosity $\mu$ for numerical calculations have been specified according to the Reynolds number of the modelled flow.

\section{Simulation of the lid-driven cavity problem}

Lid-driven cavity flow is being used as a standard test case for the validation of numerical solutions of incompressible Navier-Stokes flow. The upper wall of the cavity motions with a velocity $u=1$, and no-slip impermeable boundary conditions are considered along the other three walls. The geometry and velocity boundary conditions are demonstrated in Fig. 2. The dilemma of this problem remains in the presence of the singularities of the pressure and velocity at the two top corners of the cavity. Therefore, the amount of the computational subdomains utilized decreases towards the corners of the cavity (see Fig. 2) to catch the high pressure and velocity function gradients.

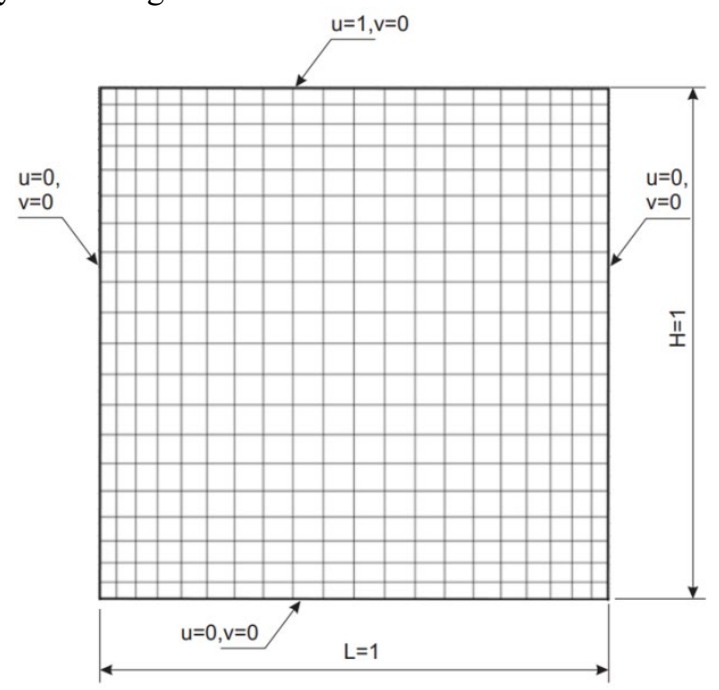

Fig. 2. The lid-driven cavity problem geometry, boundary conditions, and distribution of internal cells (subdomains).

The steady solution using the SBMDR is achieved when the discrepancy between two consecutive time steps (in the definition of the infinity norm) is lower than specified, $\varepsilon_{\mathrm{tol}}=1 \times 10^{-6}$ is applied for presented numerical computations. 

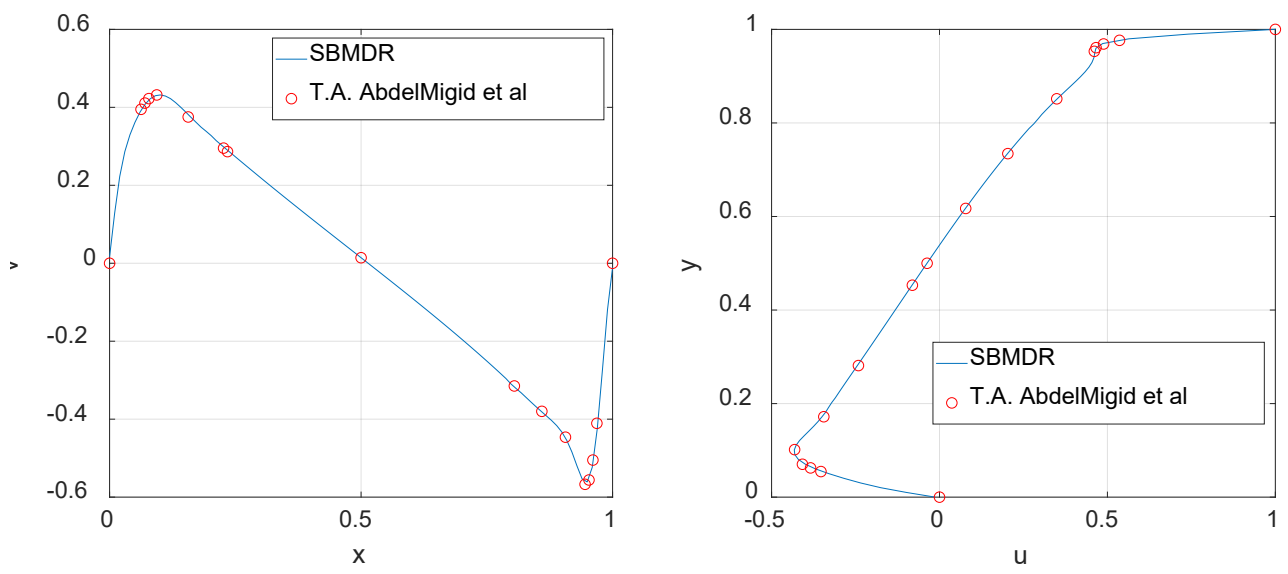

Fig. 3. Lid-driven cavity, $\operatorname{Re}=3200$, left: vertical velocity components along horizontal line $y=0.5$, right: horizontal velocity components along vertical line $x=0.5$.
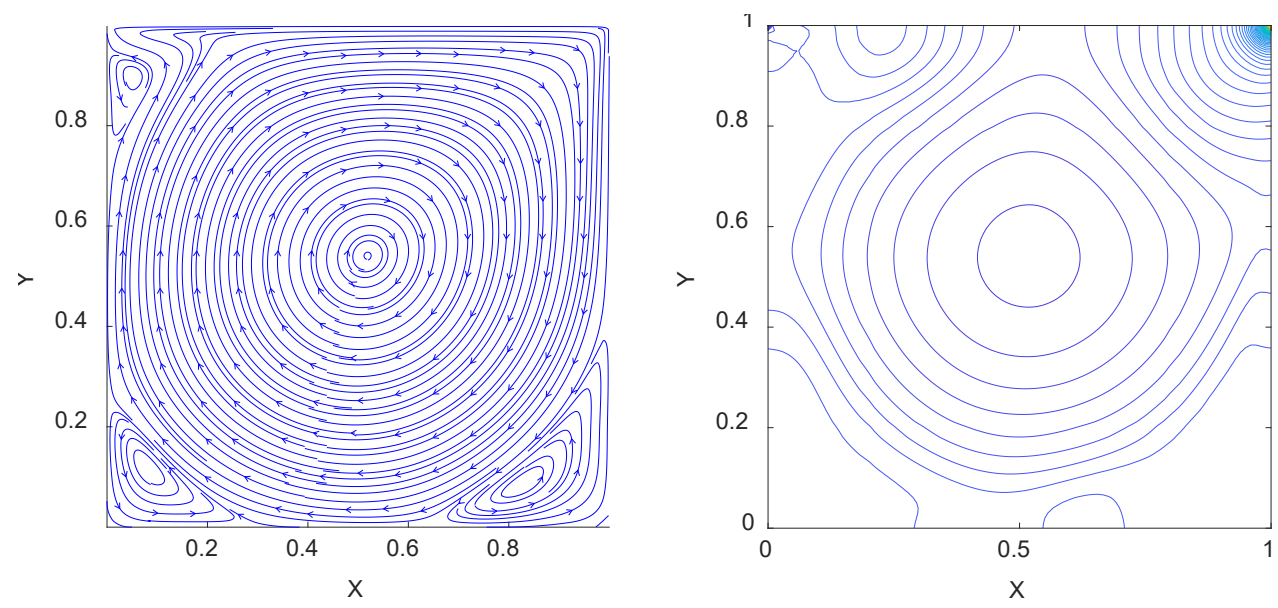

Fig. 4. Streamlines and Pressure contours for Lid-driven cavity problem, $\mathrm{Re}=3200$.

The results of the SBMDR are confronted with the solution represented in [10] for the Reynolds number $\operatorname{Re}=3200$; the vertical velocity components along a horizontal line and the horizontal velocity components along a vertical line, straight to the center of the cavity, are plotted in Fig. 3. Fig. 4 shows streamlines and pressure contours for Reynolds number of $\operatorname{Re}=3200$.

\section{Conclusions}

This paper documents the experiment to apply the global SBM and DRM (SBMDR) for the solution of the incompressible laminar flow outlined by a primitive variable form of the Navier-Stokes equation. The lid-driven cavity benchmarking problem have been successfully tested. This type of boundary collocation method seems to be a quite useful alternative to the solutions of incompressible fluid flow using the boundary-based numerical schemes. The dual reciprocity technique implemented using implicitly compactly supported RBFs 
generates a sparse characteristic matrix and enables the method to obtain reasonable results even for high Reynolds numbers. The presented numerical code and numerical results need to be more analyzed in the sense of precision, convergence and computational demands.

This contribution is the result of the project funded by the Scientific Grant Agency of Slovak Republic (VEGA) No. 1-0716-17

\section{References}

1. K. Kováŕík, D. Sitányiová, J. Mužík, Eng. Anal. Bound. Elem. 44, 55-63 (2014)

2. C.A. Brebbia, Boundary Element Techniques in Engineering. (Elsevier, 2016)

3. P. Partridge, Dual Reciprocity Boundary Element Method. (Springer Science \& Business Media, 2012)

4. M.A. Golberg, Boundary Integral Methods - Numerical and Mathematical Aspects (WIT Press, 1998)

5. K. Kovářík, J. Mužík, S. Masarovičová, D. Sitányiová, Eng. Anal. Bound. Elem. 95, 8592 (2018)

6. Šarler, B., Eng. Anal. Bound. Elem. 33, 1374-1382 (2009)

7. X. Wei, W. Chen, L. Sun, B. Chen, Eng. Anal. Bound. Elem. 58, 151-165 (2015)

8. W. Chen, Z. Fu, X. Wei, CMES-Comp. Model. Eng 54, 65-85 (2009)

9. W.F. Florez, Nonlinear flow using dual reciprocity. (WIT Press, 2001)

10. T.A. AbdelMigid, K.M. Saqr, M.A. Kotb, A.A. Aboelfarag, Alex. Eng. J. 56, 123-135 (2016) 